\title{
The metameric pattern of Hypsibius dujardini (Eutardigrada) and its relationship to that of other panarthropods
}

\author{
Frank W Smith ${ }^{1,2^{*}}$ and Elizabeth L Jockusch ${ }^{1}$
}

\begin{abstract}
Introduction: Tardigrades are an ancient lineage of microinvertebrates with a unique metameric pattern consisting of a head and four lobopodal leg-bearing segments. While their close relationship to Onychophora and Arthropoda is well established, many questions remain about the structure and origin of the tardigrade metameric pattern. For example, the relationship of the tardigrade head to that of Arthropoda and Onychophora remains a contentious issue. One source of contention stems from disagreement about the structure of the tardigrade brain. The availability of developmental tools for the tardigrade Hypsibius dujardini give this species the potential to clarify questions regarding the relationship of tardigrade segmental patterns to those in Arthropoda and Onychophora. Here we investigate the nervous system, muscle system, and cuticle anatomy of $H$. dujardini using high-resolution microscopy methods.

Results: We characterized nervous system anatomy of $H$. dujardini using a combination of anti- $\beta$-tubulin staining and DAPI staining and muscle system anatomy using phalloidin staining. We identified several brain lobes: paired outer lobes, paired inner lobes, and a single horseshoe-shaped ventral lobe. We also characterized similarities and differences in the nervous system and muscle system anatomy of the four body segments. Based on these, we detect distinct morphological identities for each segment in this species.

Conclusions: Based on comparisons of our results to previous reports, we find support for an ancestral tardigrade brain exhibiting architecture similar to that of $\mathrm{H}$. dujardini. Comparisons to other tardigrade species suggest that each segment in the ancestral tardigrade possessed a unique morphological identity, rather than exhibiting strictly homonomous segmentation, and thus that differentiation of anterior segment identities arose prior to the diversification of panarthropodan lineages. This hypothesis can be further tested by examination of the expression boundaries of anterior Hox genes, which differentiate anterior segments through a mechanism conserved between onychophorans and arthropods. Our investigation of $H$. dujardini segmental morphologies will facilitate developmental genetic studies in this species that promise to illuminate the relationship of the tardigrade metameric pattern to that of other panarthropods.
\end{abstract}

Keywords: Evolution, Homology, Tardigrada, Panarthropoda, Tagmosis

\section{Introduction}

The taxon Tardigrada is composed of microscopic invertebrates known for their remarkable ability to survive hostile environments, including extremes in temperature, pressure, salinity, radiation, and even the vacuum of outer space [1-8]. Tardigrada is an ancient lineage, with a fossil

\footnotetext{
* Correspondence: frank.w.smithiii@gmail.com

'Department of Ecology \& Evolutionary Biology, University of Connecticut, 75 N. Eagleville Road, U-3043, Storrs, CT 06269-3043, USA

${ }^{2}$ Current address: Biology Department, University of North Carolina, 616 Fordham ${ }^{2}$ Current address: Biology Department,
Hall, Chapel Hill, NC 27599-3280, USA
}

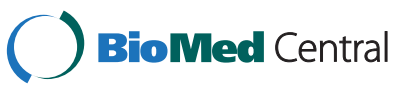

(c) 2014 Smith and Jockusch; licensee BioMed Central Ltd. This is an Open Access article distributed under the terms of the Creative Commons Attribution License (http://creativecommons.org/licenses/by/4.0), which permits unrestricted use, distribution, and reproduction in any medium, provided the original work is properly credited. The Creative Commons Public Domain Dedication waiver (http://creativecommons.org/publicdomain/zero/1.0/) applies to the data made available in this article, unless otherwise stated.

record extending back to the Cambrian period [9]. Extant tardigrade species inhabit marine, freshwater, and terrestrial habitats $[10,11]$. The metameric pattern of tardigrades consists of a head, housing a multilobed brain [12-18], and four segments bearing lobopodal legs $[10,11,19,20]$. Based on morphological similarities, a close relationship of Tardigrada with two other ancient lineages, Onychophora and Arthropoda, has long been acknowledged; the taxon Panarthropoda was erected in recognition of this relationship [10,12,20-23]. A recent taxonomically well-sampled phylogenomic analysis recovered a highly supported 
monophyletic Panarthropoda and suggested that the earliest divergence within this lineage was between Tardigrada and the ancestor of Arthropoda + Onychophora [24]. A recent analysis that included morphological data from extant and extinct species recovered the same topology [25]. However, other studies have found support for Arthropoda + Tardigrada [26] or Tardigrada + Onychophora [27].

Both arthropods and onychophorans exhibit clear head tagmata $[10,28,29]$, which house the brains of these animals. In one model of panarthropod nervous system evolution, ancestrally each segment housed a ganglion [30]. In this model, the three parts of the arthropod brain, the protocerebrum, deutocerebrum, and tritocerebrum, are derived from ancestral ganglia of the anterior three segments. Onychophorans possess a head tagma composed of only three morphologically and functionally distinct segments, and a trunk composed of many morphologically similar segments [10]. The onychophoran brain is derived from nervous tissue of the two anterior-most segments [31]. Fossil evidence that the common ancestor of arthropods and onychophorans lacked tagmosis [32,33], along with differences in tagmosis patterns in arthropods and onychophorans, suggest that tagmosis evolved independently in these two lineages [27].

The relationship of the tardigrade head to the heads of arthropods and onychophorans has important implications for the evolution of tagmosis in Panarthropoda. However, how the segments of tardigrades align to those of arthropods and onychophorans is a contentious issue. This debate stems in part from disagreements concerning the relationship of the tardigrade brain to the arthropod brain. In the tripartite hypothesis of tardigrade brain composition, paired outer, inner, and ventral brain lobes are considered to be serially homologous to segmental ganglia [17-20,34]. This is based on similarities in structure between lobes and ganglia, such as the presence of commissures. Under this hypothesis, there is direct homology between tardigrade brain lobes and the three segments of the arthropod brain [17-20,34]. Generally, the inner lobe of the tardigrade brain is aligned with the protocerebrum, the outer lobe with the deuterocerebrum, and the ventral lobe with the tritocerebrum [20,34]. There are several important evolutionary implications of this hypothesis. If the tardigrade brain is composed of three ancestral segmental ganglia, then the tardigrade head originated by fusion of three ancestral segments. Tardigrades would possess a head tagma under this hypothesis. Under the hypothesis that Onychophora is the sister group of Arthropoda, it suggests that the bipartite brain of Onychophora is derived from an ancestral tripartite brain. Alternatively, if tardigrades are the sister group of arthropods, then the tripartite brain may be a synapomorphy of this clade. By contrast, the protocerebral hypothesis of brain composition asserts that the entire tardigrade brain is directly homologous to the protocerebrum of arthropods [15,35], and therefore that the tardigrade head is composed of a single segment and the tardigrade brain is derived from the ganglion of that segment. Under this hypothesis, the tripartite brain is a synapomorphy of Arthropoda [31].

Proponents of the protocerebral hypothesis have presented high-resolution confocal microscopy investigations of brain anatomy of two species of Macrobiotus (Eutardigrada) [15,35], Echiniscus testudo (Heterotardigrada) [36], Batillipes pennaki (Heterotardigrada) [37], and Actinarctus doryphorus ocellatus (Heterotardigrada) [37]. Proponents of the tripartite hypothesis have presented confocal microscopy investigations of Halobiotus crispae (Eutardigrada) [17] and Actinarctus doryphorus (Heterotardigrada) [18]. The above studies are generally in agreement that tardigrades possess a multilobed brain, but how many lobes are present in the brain and the relationship of brain lobes across Tardigrada are not well resolved. Moreover, investigations of brain lobes and commissures alone have been unable to resolve the relationship of the tardigrade brain to that in other panarthropods.

Several additional aspects of anterior nervous system anatomy have been evaluated in attempting to homologize tardigrade brains to those of other panarthropods. According to the tripartite hypothesis of tardigrade brain architecture, an arthropod-like subesophogeal ganglion is also present in the tardigrade head. By contrast, under the protocerebral hypothesis, there is no subesophogeal ganglion, because its existence would require the brain to be composed of at least two ganglia. A subesophageal ganglion was included in the brain anatomy interpretations of several studies $[13,17,18,20,34]$. However, most recent studies of tardigrade brain anatomy have not identified a subesophogeal ganglion [15,35-37]. In arthropods, the stomatogastric ganglion is innervated by the tritocerebrum [38]. Mayer et al. [15] interpreted a structure above the ventral ganglion of the second leg-bearing segment of Macrobiotus harmsworthi as a stomatogastric ganglion, suggesting that the third segment of $M$. harmsworthi aligns with the tritocerebral segment of arthropods, and by extension, that the brain-housing segment of M. harmsworthi aligns with the protocerebrum of arthropods [15]. However, a candidate stomatogastric ganglion has not been identified in other tardigrade species.

One explanation for these conflicting conclusions is that there is variation in brain anatomy across Tardigrada. Alternatively, the putative differences may instead reflect different evolutionary interpretations of similar brain morphologies by workers in the field. Although it seems possible that nervous system anatomies have diverged among tardigrade lineages, segmental patterns of muscle anatomy are highly conserved across tardigrades [39], suggesting that, at the very least, all tardigrades 
share the same complement of directly homologous segments.

The value of developmental studies for discriminating between the protocerebral and tripartite hypotheses has been acknowledged by proponents of both interpretations of tardigrade brain morphology [15-17,35,37]. Such data have put to rest long-standing controversy regarding the composition of the chelicerate head [40-42]. The tardigrade Hybsibius dujardini (Parachela, Eutardigrada) is an ideal system for testing these and other hypotheses related to the evolution of tardigrades with developmental data. First, $H$. dujardini is easily cultured, providing unlimited access to embryos for experimentation [43]. Second, the feasibility of embryonic studies of gene expression $[44,45]$ and gene function through RNAi [46] has been demonstrated for this species. Third, an $H$. dujardini genome sequencing project is in progress (Goldstein, pers. comm.). Mayer et al. [16] previously investigated the organization of the nervous system of $H$. dujardini. In order to investigate segmental patterns in $H$. dujardini in further detail, we stained the nervous system with a $\beta$-tubulin antibody and the muscle system with phalloidin, and then analyzed the segmental structure of these systems with confocal microscopy. We also examined the cuticle of $H$. dujardini using scanning electron micrographs. Our results reveal that $H$. dujardini retains highly conserved segmental muscle and nervous system anatomies, supporting the suitability of this species as a developmental model for Tardigrada. Our results support an ancestral tardigrade brain composed of paired outer lobes, paired inner lobes, and a ventral lobe. We propose that the ventral lobe of $H$. dujardini is directly homologous to both the ventral lobe and subesophogeal ganglion identified in previous studies $[17,18]$. Furthermore, these results suggest that internal morphological aspects of the anterior segments were already differentiated in the panarthropod ancestor. Thus, although the panarthropod common ancestor is characterized as being homonomously segmented [19,22,23,32,47-50], these comparative data suggest that segmental diversity may have evolved prior to the divergence of the three panarthropod lineages. The developmental mechanism responsible for differentiating anterior segments in Onychophora and Arthropoda is conserved [51,52]. The discovery of segmental diversity in tardigrades raises the question of whether this mechanism is even evolutionarily more ancient.

\section{Results}

\section{General morphology}

Like other tardigrades, $H$. dujardini possesses a head and four trunk segments. A pair of ventral legs is found in each trunk segment (Figure 1A). A series of claws attach to the distal tip of each leg (c1-c4, Figure 1A, D).
The head lacks legs, but has an internal pharyngeal apparatus, which has been suggested to be derived from an ancestral pair of head appendages [20,53]. The pharyngeal apparatus includes a muscular pharyngeal bulb $(\mathrm{ph})$ and stylets (st), which are used in feeding (Figure 1C). The mouth is found at the anterior end of the head (mo, Figure 1A). Below we describe the nervous and muscular systems of H. dujardini. We identify nervous system anatomy and muscle system anatomy with terminology based on previous confocal microscopy analyses $[15,17,35,53,54]$.

\section{Nervous system anatomy}

We used a combination of DAPI-staining and anti- $\beta$ tubulin immunohistochemistry to characterize the nervous system of $H$. dujardini. The $H$. dujardini nervous system is composed of an anterior brain, a pair of ventral nerve cords, and a peripheral nervous system composed of many smaller nerves. In DAPI-stained specimens, we identified several clusters of somata in the head, which we refer to as brain lobes. Two bilaterally symmetrical pairs of brain lobes, outer lobes (ol) and inner lobes (il), were detected, along with a ventral lobe (vl) that spanned the midline (Figure $2 \mathrm{~B}-\mathrm{E}$ ). In anti- $\beta$-tubulin stained specimens, two nerves, the outer connective (oc) and the inner connective (ic), could be seen extending from both the right and left anterior region of the first trunk ganglion into the brain (Figure 2A). The outer connective extends dorsally to the dorsal cluster (dc) near the eye. The inner connective extends through the ventral lobe (vl) into a region of dense nervous tissue (ne) innervating the mouth (Figures 2A and 3A). Anterior clusters (ac) of nerves innervate the papillae cephalica (Figures 2A and 3A, D). A dorsal commissure (dco) connects the outer brain lobes (Figure 2A). An inner commissure (ico) connects the inner brain lobes (Figure 2A). A commissure $\left(\mathrm{co}^{*}\right)$ also extends between the inner connectives beneath the ventral lobe (Figure 3A, C, E). Several nerves extend posteriorly from the brain along the sides of the pharyngeal bulb (labeled 'pn' in Figures 2A and 3D).

Four ganglia are found along the ventral nerve cords, one for each trunk segment (ga1-ga4, Figure 3A). Each ganglion includes a single dense cluster of cell bodies that does not show anti- $\beta$-tubulin expression. The ventral nerve cords extend ventrolaterally across the cell clusters. Within each ganglion, the ventral nerve cords swell to roughly three times the width of the connectives between the ganglia. The ventral nerve cords are connected by fibrous commissures that extend across the cell cluster of each ganglion (co, Figure 3A, C-E, G). Nerves extend from each ganglion into the legs of the segment in which the ganglion is found. The first three ganglia are wider than the fourth ganglion (Figure 3A, G). For example, in the specimen shown in Figure 3A, from ganglion 1 to 4 , the maximum widths of the ganglia 

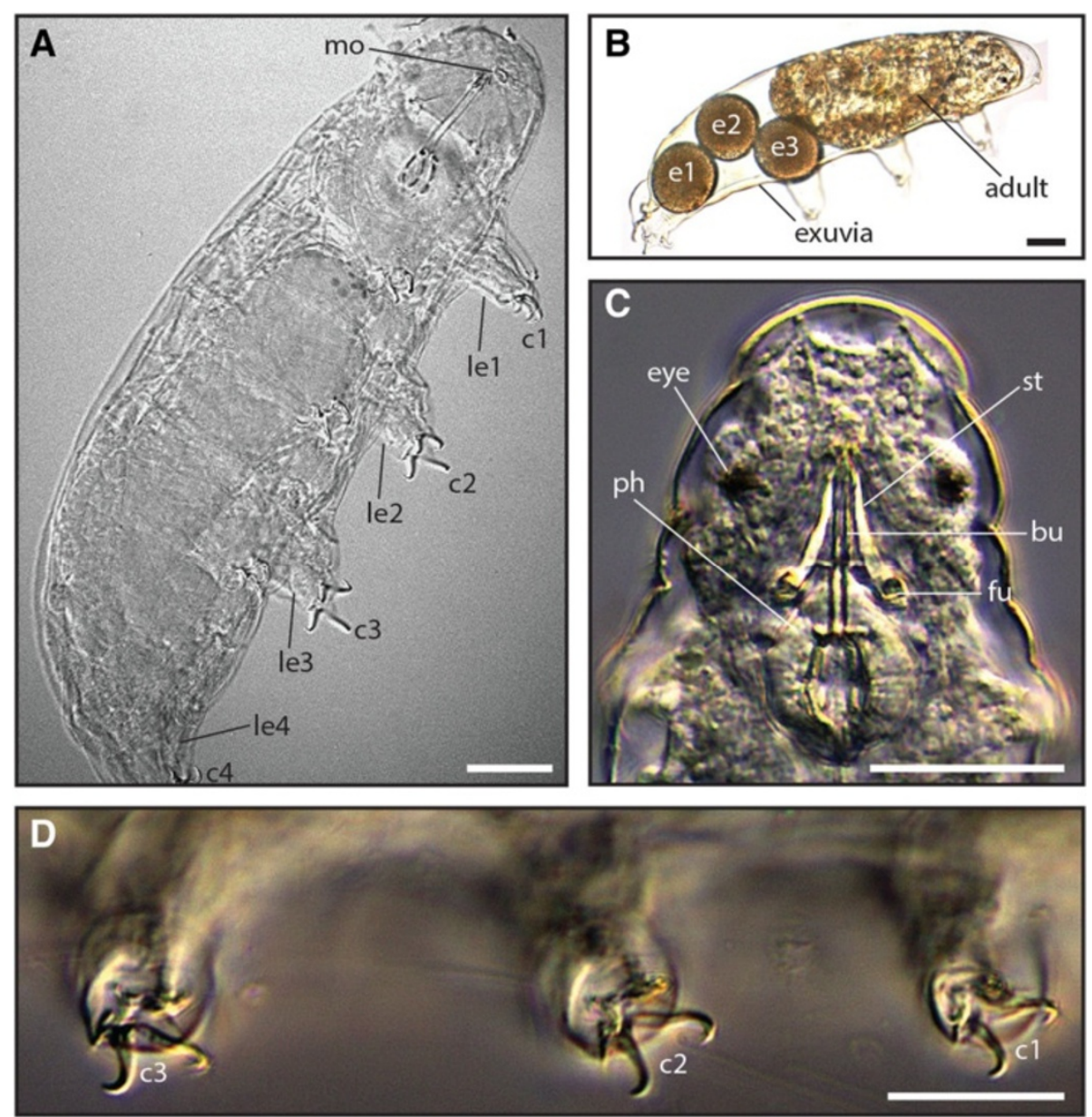

Figure 1 General anatomy of $\boldsymbol{H}$. dujardini. Scale bars equal $25 \mu \mathrm{m}$ (A) Differential interference micrograph showing ventrolateral view of the left side of an adult $H$. dujardini specimen. The anteroposterior axis includes a head and four trunk segments, each with a pair of legs (left legs are in focus). (B) Light micrograph of an adult laying eggs in its exuvia during molting. (C) DIC micrograph of the ventral head of an adult $H$. dujardini specimen. (D) DIC micrograph of the leg claws of the anterior three right legs. Abbreviations: bu, buccal tube; C, claw; e, eggs; fu, furca; le, leg; mo, mouth; ph, pharyngeal bulb; st, stylet.

measured 12.4 $\mu \mathrm{m}, 12.8 \mu \mathrm{m}, 13.4 \mu \mathrm{m}$, and $8.0 \mu \mathrm{m}$, respectively. Anti- $\beta$-tubulin expression in the stomach in Figure $3 \mathrm{~A}$ and $\mathrm{G}$ is autofluorescence due to algal gut content (see Additional file 1: Figure S1).

\section{Ventral and lateral musculature}

Muscles were visualized using phalloidin staining. A pair of bilaterally symmetric muscle strands extends longitudinally throughout much of the ventral axis (ve, Figure 4A). Each of these muscle strands is interrupted by 7 intermediate attachment sites (a-g, Figures $4 \mathrm{~A}$ and $5 \mathrm{~A}$ ). At its corresponding attachment site $\mathrm{c}$, each muscle strand bifurcates and extends in an anterior direction past the pharyngeal bulb. For both ventral longitudinal muscles, one strand of each bifurcation extends to intermediate attachment site $\mathrm{a}$, and the other to intermediate attachment site $\mathrm{b}$ (Figure 5A).
Seven ventral median muscle attachment sites are found in $H$. dujardini and other tardigrade species (1-7, Figure 4A). These attachment sites are visible as depressions in the ventral cuticle of $H$. dujardini (Figure 4B). Muscles attaching at the ventral median attachment sites extend to the base of the legs or into the legs, run along the ventral muscle strands, extend dorsoanteriorly, extend anteriorly along the ventral muscle bands, or run dorsally to lateral muscle attachment sites. Lateral muscle attachment sites are also visible as depressions in the exterior cuticle (Figure 5B). We label lateral muscle attachment sites following the scheme of Halberg

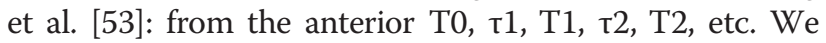
analyzed the structures of the ventral attachment sites in seven phalloidin-stained $H$. dujardini specimens; morphology of ventral median attachment sites was consistent across specimens. All ventral median attachment sites are 

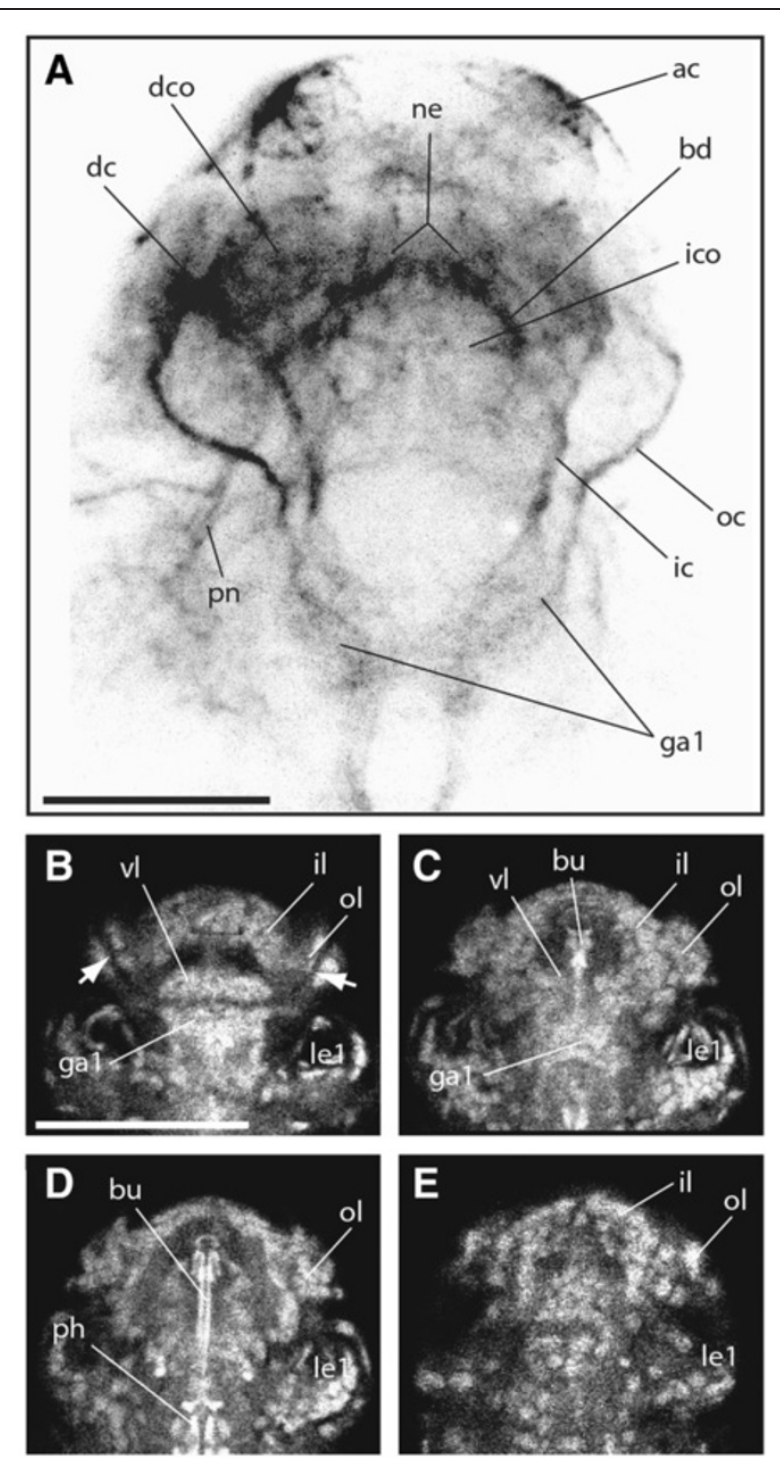

Figure 2 Structure of the $\boldsymbol{H}$. dujardini head. All images are of ventral-mounted specimens. Scale bar equals $25 \mu \mathrm{m}$. (A) Maximum projection of confocal Z-series. (B-D). Increasingly deeper slices of a DAPI-stained specimen. (B) The ventral lobe appears continuous across the midline. Note the groove that runs through the outer (arrows) brain lobes. (C) The ventral lobe extends on both sides of the buccal tube, suggesting that it is horseshoe shaped. (D) Few cell bodies surround the buccal tube in the interior of the head. (E) Dorsal brain. Abbreviations: ac, anterior cluster; bd, neurite bundles; bu, buccal tube; dc, dorsal cluster; dco, dorsal commissure; ga, ganglion; ic, inner connective ico, inner commissure; il, inner brain lobe; le, leg; ne, neurites of the mouth; oc, outer connective; ol, outer brain lobe; ph, pharyngeal bulb; pn, posterior nerves; vl, ventral brain lobe.

bilaterally symmetrical, and unless indicated below, muscles they support do not cross the midline. Here we provide anatomical details for one side of each ventral attachment site.
The first ventral median attachment site includes 7 muscle pairs that do not make contact medially (Figure 4A inset). Muscle strand 1a at this site extends anteriorly to an intermediate attachment site (a) of the ventral longitudinal muscle strand. Muscle strand 1i extends into the first leg. A thin muscle strand ( $\left.1 \mathrm{i}^{*}\right)$ extends across the midline into the first leg of the other side. This is the only instance of a ventral muscle strand crossing the midline. Two thin muscle strands (1ii) extend dorsally to lateral longitudinal attachment site T0 (Figure 5A). Next, two thick muscle strands (1iii) extend to the base of the second leg (Figure 4A, see leg muscles section below).

The second ventral median attachment site includes three pairs of muscles that make contact medially (Figure 4A). The anterior-most muscle strand (2i) extends into the first leg, where it forms a sheet muscle along the poster wall of the leg (see leg muscles section). Next a bifurcated muscle strand (2ii) extends dorsally along the body wall to lateral longitudinal attachment site $\tau 1$ (Figures 4A and 5A). The posterior-most muscle extends to the base of the second leg (2iii, Figure 4A). The third ventral median attachment site includes five muscle pairs that do not make contact medially (Figure 4A). The anterior-most muscle strand (3a, Figure 4A) extends anteriorly to an intermediate attachment site (c) of the ventral longitudinal muscle strand. This muscle is followed by a muscle strand that extends into the second leg (3i). Two thin muscle strands (3ii) extend dorsally along the body wall to longitudinal attachment site T1 (Figures 4A and $5 \mathrm{~A}$ ). These are followed by a muscle strand that extends to the base of the third leg (3iii, Figure 4A).

The architecture of ventral median attachment site 4 is identical to that of attachment site 2 (Figure 4A). In this case, the anterior-most muscle strand (4i) runs into the second leg, muscle strand 4ii extends to longitudinal attachment site $\tau 2$, and the posterior-most muscle strand (4iii) runs to the base of third leg (Figures 4A and 5A). Ventral median attachment site 5 is similar to attachment site 3. In this case, muscle strand (5i) extends from attachment site 5 laterally into the third leg and two thin muscle strands (5ii) extend to longitudinal attachment site T2 (Figures $4 \mathrm{~A}$ and $5 \mathrm{~A}$ ). Attachment site 5 differs from attachment site 3 in that the anterior-most muscle strand (5a) runs dorsoanteriorly to a lateral position (Figure 5A) and continues in an anterior direction and no posteriorly-directed muscle strand is present.

Two muscle pairs are attached to ventral median attachment site 6 (Figure 4A); these come into contact medially. The anterior muscle strand (6i) extends laterally into the third leg and forms a sheet muscle along the posterior of the leg (see leg muscles section). The posterior muscle strand (6ii) splits into two muscle bands, which run dorsally along the body wall to longitudinal attachment site $\tau 3$ (Figure 5A). Four muscle pairs that do not make contact medially extend from ventral 

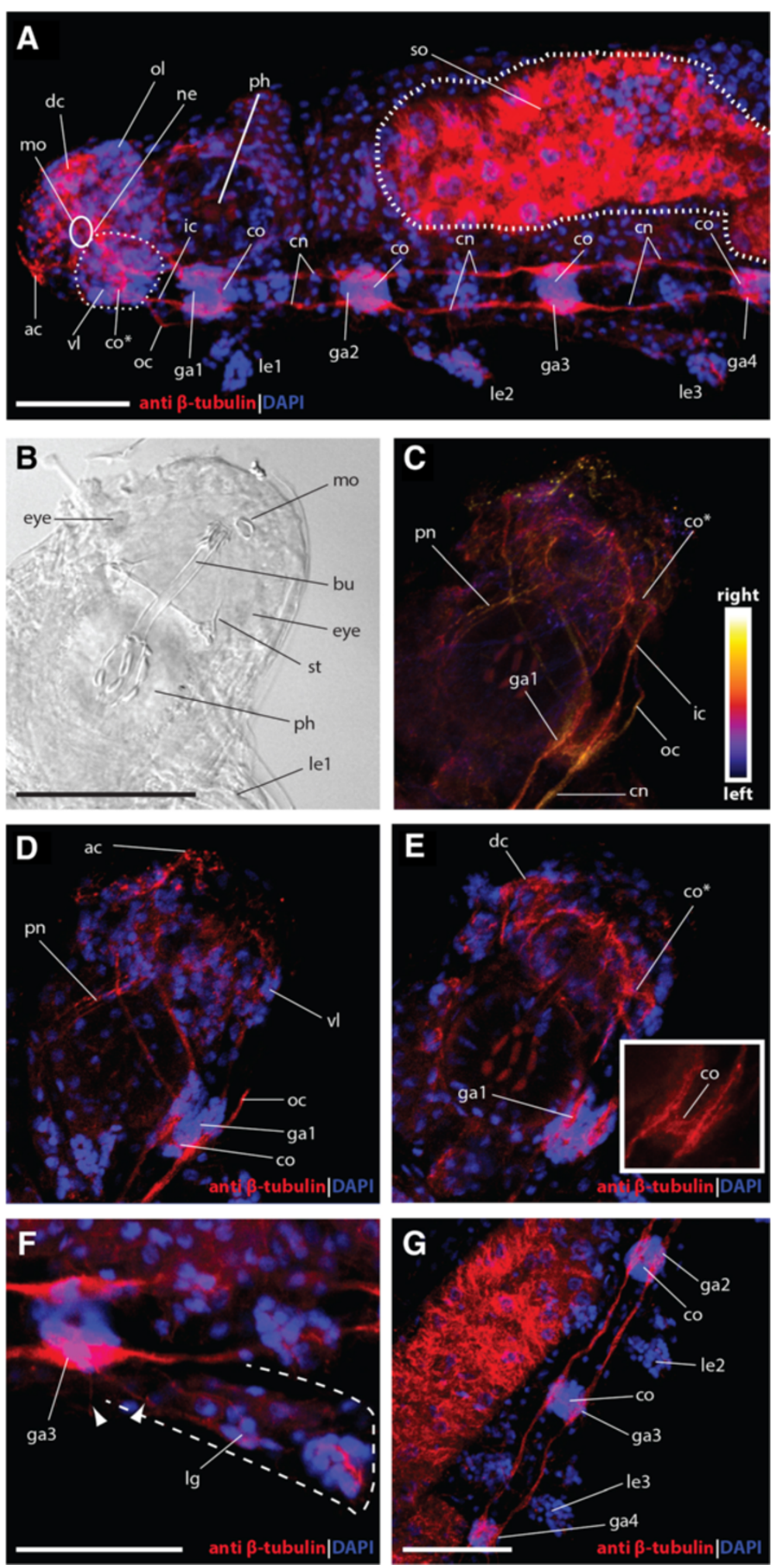

Figure $\mathbf{3}$ (See legend on next page.) 
(See figure on previous page.)

Figure 3 "Rope ladder-like" ventral nervous system of $\boldsymbol{H}$. dujardini. Anti- $\beta$-tubulin immunolabeling is shown in A, C-G. DAPI counterstaining is shown in A, D-G. Scale bars equal $25 \mu \mathrm{m}$. (A) Maximum projection of brain and ventral nervous system. Each body segment includes a ventral ganglion. The inner connective extends from the anterior-most ventral ganglion to the ventral brain lobe, where a commissure (co*) transverses the midline. The position of the mouth is indicated. (B) DIC micrograph of the head. (C) Depth-coded maximum-projection of confocal Z-series of specimen shown in B. The brain and first ganglion can be seen. (D-E) Individual slices of the Z-series used to produce $\mathbf{C}$. Inset in $\mathbf{E}$ shows a maximum projection magnification of the ganglion; fibrous commissures extend across the ganglion. (F) Maximum projection of the ganglion and a leg of the third trunk segment (same specimen as shown in $\mathbf{A}$ ). The right leg is outlined. Arrowheads point to nerves extending from the third ganglion. (G) Maximum projection of the second, third, and anterior fourth segments (same specimen as shown in B-E). Ganglion 4 is smaller than ganglion 2 or 3 . Abbreviations: ac, anterior cluster; bu, buccal tube; cn, connective; co, commissure; dc, dorsal cluster; ga, ganglion; ic, inner connective; le, leg; lg, leg ganglion; mo, mouth; ne, neurites of the mouth; oc, outer connective; ol, outer lobe; ph, pharyngeal bulb; pn, posterior nerves; so, stomach; st, stylet; vl, ventral lobe.

median attachment site 7 (Figure 4A). The anterior muscle strand (7a) extends dorsoanteriorly to a lateral position and continues in an anterior direction (Figure 5A). Two thin muscle strands (7ii) extend dorsally along the body wall to longitudinal attachment site T3 (Figure 5A). Finally a muscle strand (7iii) extends to the base of the posteriormost leg (Figure 4A).

\section{Dorsal musculature}

Two pairs of longitudinal dorsal muscle strands extend from in front of the pharyngeal bulb (ph) along the dorsum to the posterior end (Figure 6A). Several muscle attachment sites are found along the dorsal muscle strands, which by convention are given capital letter designations. The outer strand of each pair extends from attachment site A to attachment site W. The inner strand extends from attachment site $A^{*}$ to dorsal attachment site $\mathrm{V}$. Longitudinal muscle stands of each pair appear to fuse and split at several positions along their lengths. Several muscle attachment sites are located along these muscle strands. Muscles extending from near the pharyngeal bulb attach to the outer longitudinal muscle strand at attachment sites B and C. At the next several attachment sites, the longitudinal muscle strands connect to muscles extending
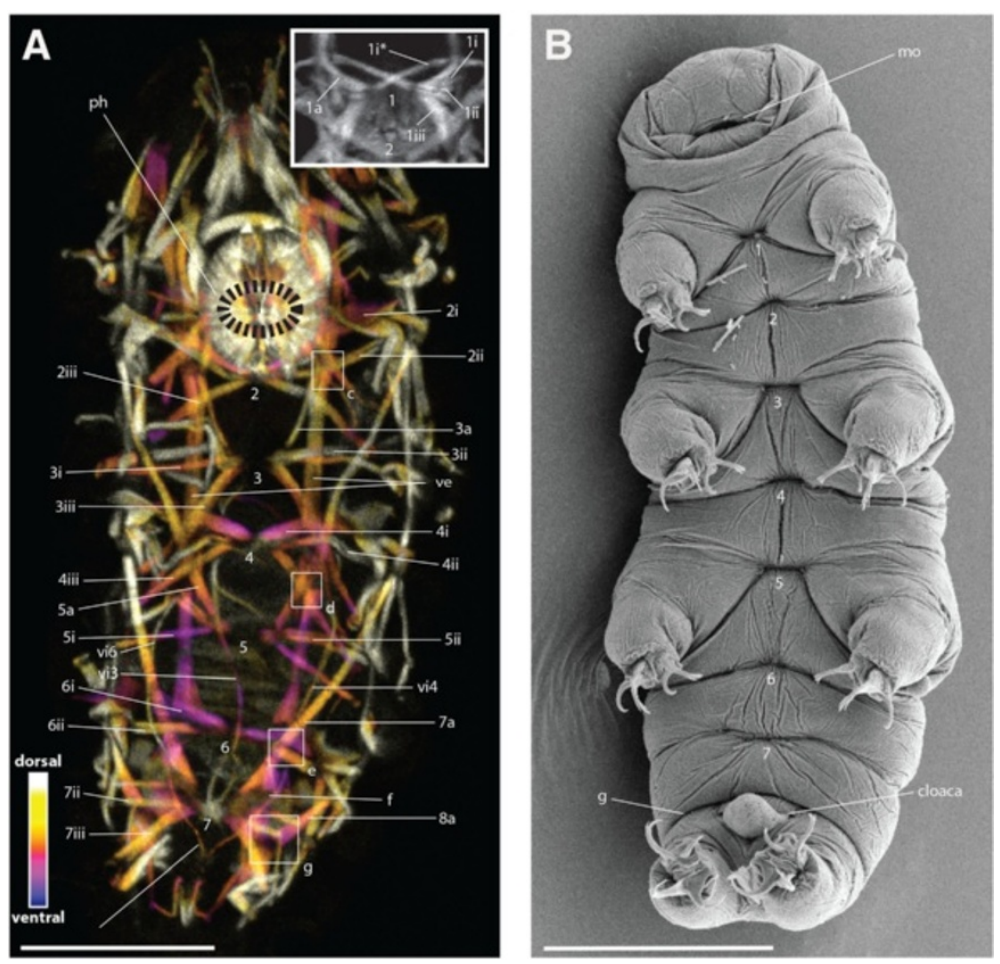

Figure 4 Ventral muscle anatomy in $\boldsymbol{H}$. dujardini as revealed by phalloidin staining. Specimens are oriented ventral side up. Anterior is to the top. Scale bars equal $25 \mu \mathrm{m}$. (A) Depth-coded maximum projection of ventral slices of confocal Z-series. Ventral median attachment sites are numbered 1-7. Muscle strands are labeled following the system of Schmidt-Rhaesa and Kulessa [54]. The first attachment site is out of focus below the pharyngeal bulb. We have outlined its position. Inset shows a maximum projection of the first attachment site. Intermediate attachment sites along one ventral longitudinal muscle strand are boxed. (B) Scanning electron micrograph showing cuticular depressions associated with ventral attachment sites. Abbreviations: mo, mouth; ph, pharyngeal bulb; ve, ventral longitudinal muscle; vi, visceral muscle. 

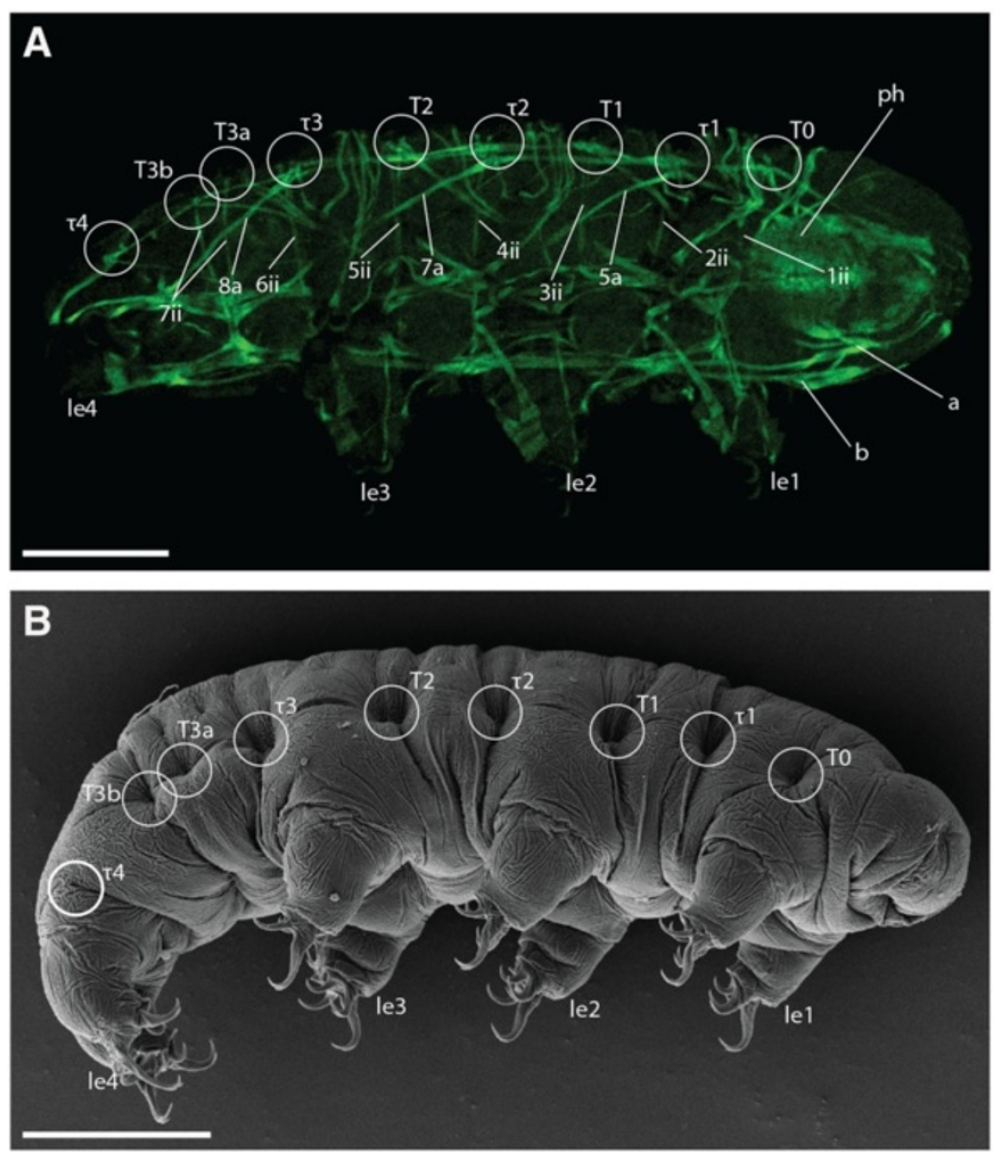

Figure $\mathbf{5}$ Lateral muscle anatomy of $\boldsymbol{H}$. dujardini as revealed by phalloidin staining. Lateral muscle attachment sites are labeled following the system of Halberg et al. [54]. Anterior is to the right. Scale bars equal $25 \mu \mathrm{m}$. (A) View of longitudinal musculature. Lateral attachment sites are circled. Muscles labeled 'ii' extend from ventral attachment sites to longitudinal attachment sites. Intermediate attachment sites $a$ and $\mathrm{b}$ are visible in the ventral anterior and muscles labeled 'a' attach to these. (B) Scanning electron micrograph showing cuticular depressions associated with longitudinal attachment sites. Abbreviation: le, leg; ph, pharyngeal bulb.

from the lateral region of the legs (see leg muscles section). Attachment sites D, E, H, I, N, O, S, U and W are on the outer dorsal muscle strand. Attachment sites F, G, K, L, P and $\mathrm{Q}$ are connected to leg muscles that cross over the outer longitudinal muscle strand and connect to the inner stand. $\mathrm{F}^{*}, \mathrm{~L}^{*}$ and $\mathrm{Q}^{*}$ represent dorsal attachment sites that are off of the longitudinal muscle stands, but connect to leg muscles like most other attachment sites. Attachment sites $\mathrm{M}$ and $\mathrm{R}$ are points where longitudinal muscle strands bifurcate. Dorsal attachment points are visible as depressions in the dorsal cuticle of $H$. dujardini and cause folding of the cuticle (Figure 6B).

\section{Visceral musculature}

The visceral musculature of tardigrades functions in food acquisition and digestion. The muscular pharyngeal bulb (ph, Figures 4A, 5A and 6A) of $H$. dujardini sits above the ganglion of the first leg-bearing segment (Figure $3 \mathrm{~A}$ ). The stylets (st) sit in front of the pharyngeal bulb (Figure 1C). A total of 10 muscles are attached to the stylets of the pharyngeal apparatus near the dorsoanterior region of the phanyngeal bulb (Figure 6A). 2 muscle pairs $(\mathrm{m} 1, \mathrm{~m} 6)$ that extend from the furca (fu) to the anterior end of the buccal tube, 2 muscle pairs $(\mathrm{m} 2, \mathrm{~m} 3)$ that extend from the pharyngeal bulb to the furca, and 2 muscles $(\mathrm{m} 4, \mathrm{~m} 5)$ that extend from the furca of the left stylet to the furca of the right stylet. We detected an additional six visceral muscles in the gut cavity (vi1-6, Figures 4A and 6A), which likely function in digestion.

\section{Leg muscles}

In terms of leg musculature, the first three pairs of legs are much more similar to each other than they are to the fourth pair of legs. The medial muscles of the first three leg pairs are connected to ventral median attachment sites and are labeled as such (Figure 7A). Each of these legs includes a posterior sheet muscle (sh) that extends from the ventral median attachment site just posterior to it (2i, $4 \mathrm{i}, 6 \mathrm{i})$. Each of these legs also includes a muscle that extends to its distal tip from an adjacent 

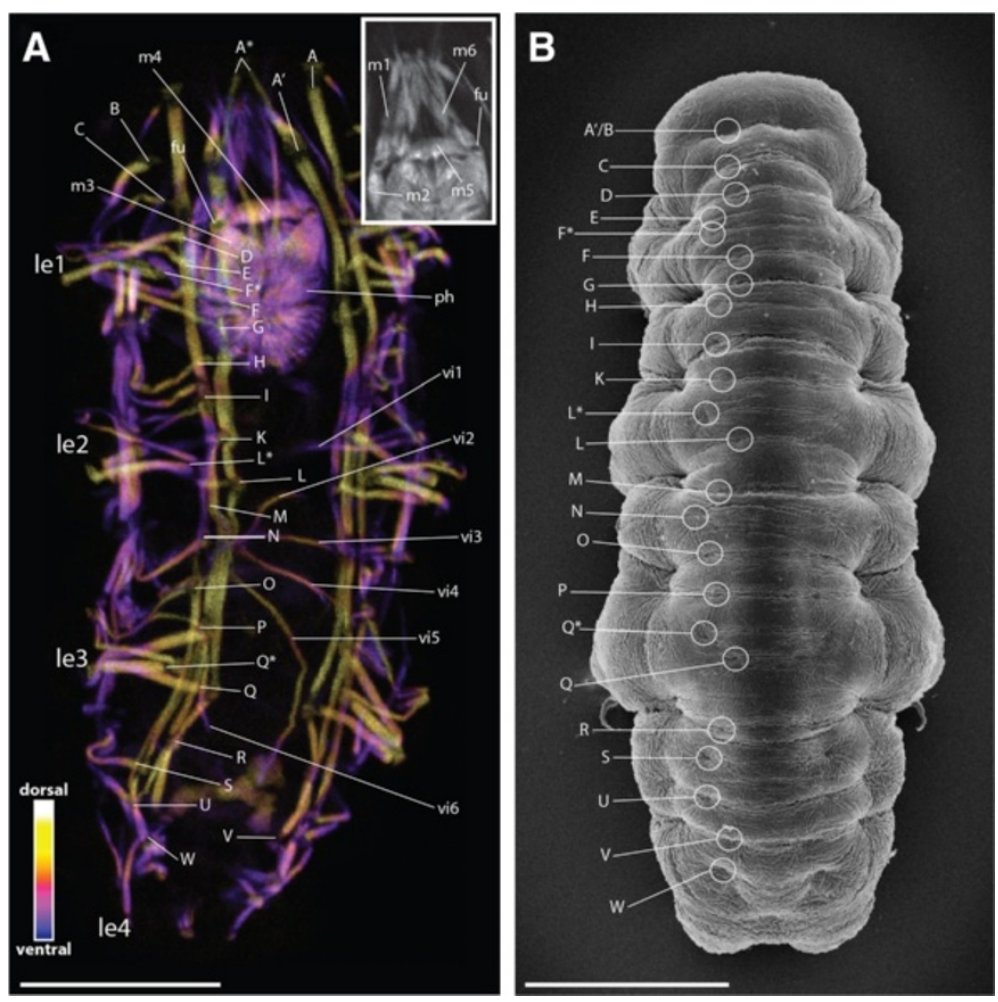

Figure 6 Dorsal muscle anatomy and visceral musculature of $\boldsymbol{H}$. dujardini as revealed by phalloidin staining. Anterior is to the top. Scale bars equal $25 \mu \mathrm{m}$. (A) Depth-coded maximum projection of dorsal slices of confocal Z-series. Same specimen as in Figure 4A. Dorsal attachment sites are labeled with capital letters. Inset shows a maximum projection of stylet muscles not visible in the depth-coded image. (B) Scanning electron micrograph showing cuticular depressions and wrinkles associated with dorsal attachment sites. Abbreviations: fu, furca; le, leg; ph, pharyngeal bulb; vi, visceral muscle.

ventral median attachment site (1i, 3i, 5i). Muscles of the iii variety attach to leg pairs two (1iii, 2iii) and three (3iii, 4iii) from the corresponding ventral attachment sites anterior to these legs (Figure 7A). No iii muscles extend to the first leg pair. Unlike the other leg pairs, a muscle extends across ventral attachment site (1) between the first leg pair into the contralateral leg (1i). For the lateral leg, muscles are named after the attachment sites they extend to. As with the medial muscle patterns, there are similar lateral muscle patterns between the legs of the first three leg pairs (Figure 7B, C). Each leg of the first three leg pairs includes a muscle $(\mathrm{t} 1, \mathrm{t} 2, \mathrm{t} 3 \mathrm{~b}$, respectively) that extends in a dorsoposterior direction to a lateral attachment site. Each leg of the first three leg pairs also includes a muscle that extends dorsally to a lateral attachment site $(\tau 1, \tau 2, \tau 3$, respectively). Finally, each of these legs also includes several muscles that extend to dorsal longitudinal attachment sites, including two muscles, each (f*1 and $2,1^{*} 1$ and 2 , $\mathrm{q}^{* 1} 1$ and 2, respectively), that extend to dorsal attachment sites that are off of the dorsal longitudinal muscle strands. In contrast to the three anterior leg pairs, very few muscles extend into the posterior-most legs (Figure 7C). A ventral longitudinal muscle strand extends into each leg of this pair, where it forms a sheet muscle (sh). A muscle (7iii) extends from each posterior leg to ventral attachment site 7 . Finally, a muscle extends from each posterior leg (w1) to dorsal attachment site W.

\section{Discussion}

\section{Segmental patterns in $\mathrm{H}$. dujardini}

Based on muscle and nervous system anatomy, we detected five segments in $H$. dujardini, each exhibiting a distinct morphological identity (summarized in Figure 8A). Characterization of distinct identities is valuable for developmental analyses and also informs our understanding of panarthropod and tardigrade evolution. The head is distinct, with the presence of the brain, stylets, and stylet musculature. In our model of segmentation, a pair of legs and the associated ganglion define each segment posterior to the head; ventral attachment sites are considered to be part of the same segment as the adjacent ganglion. Attachment site 1 is part of the first leg-bearing segment, along with the first ventral ganglion. Attachment site 1 exhibits a unique morphology compared to other ventral attachment sites, and the first leg-bearing segment is unique in housing just a single attachment site. The arrangement of leg muscles in the first leg segment was also unique. 
Figure 7 Side-mounted $H$. dujardini specimen showing leg muscle anatomy as revealed by phalloidin staining. Anterior is to the right. Scale bar equals $25 \mu \mathrm{m}$. (A) Maximum projection of the medial muscles of the left three anterior-most legs. All medial leg muscles extend to ventral median attachment sites. Ventral median attachment sites are numbered 1-7. Right legs are outlined. (B) Maximum projection of the lateral muscles of the left three anterior-most legs. Lateral leg muscles extend to dorsal attachment sites (lower case letters correspond to letter designation of dorsal attachment site) or to lateral attachment sites (muscles labeled $t, \tau$ ). (C) Maximum projection of the lateral (right leg) and medial (left leg) muscles of the posteriormost legs. Dashed lines in the posterior denotes boundary between legs and body wall. A dashed line also outlines the right third leg. The posterolateral muscle strands of the third leg are labeled (u1, s1). Dorsolaterally directed muscle 8a is labeled. Abbreviations: ga, ganglion; le, leg; sh, sheet muscle; ve, ventral longitudinal muscle strand.

Attachment sites 2 and 3 belong to the second leg-bearing segment. These attachment sites circumscribe the ventral ganglion of this segment (ganglion 2). Attachment sites 4 and 5 are parts of the third trunk segment, and circumscribe ganglion 3. Attachment site 4 shares an identical muscle arrangement with attachment site 2, while the anterior of attachment site 5 is similar to the anterior of ventral attachment site 3. Segments 2 and 3 share leg muscle architecture as well as attachment site architecture. However, they are distinguished by the muscle arrangement in the posterior of attachment sites 3 and 5. Attachment sites 6 and 7 are parts of the fourth trunk segment; the morphologies of both are unique compared to those of other attachment sites. These sites circumscribe ganglion 4 . The ganglion of this segment is the smallest of the segmental ganglia. Based on muscle anatomy in the legs, we detected three distinct leg identities. Both appendage pairs one and four have distinct morphologies, while appendage pairs two and three are nearly identical. Therefore, while we could detect distinct segmental identities among the legbearing segments, based on morphology, it is clear that leg-bearing segments 2 and 3 are the most similar. Below, we compare our results to those from other tardigrade species to reconstruct the antiquity in Tardigrada of the segmental patterns we detected in $H$. dujardini.

\section{Nervous system anatomy of $H$. dujardini and its} relationship to that of other tardigrade species

The tardigrade brain is composed of several lobes, which are at least party composed of neuronal somata [15,16,18,35-37]. The brain of $H$. dujardini is composed of two paired lobes (outer and inner) and one unpaired lobe (ventral). Outer and inner lobes have been identified in brains across Tardigrada $[15,17,18,35,36]$. The similar location of these brain structures across Tardigrada supports their direct homology in this lineage. Ventral lobes and a subesophogeal ganglion 


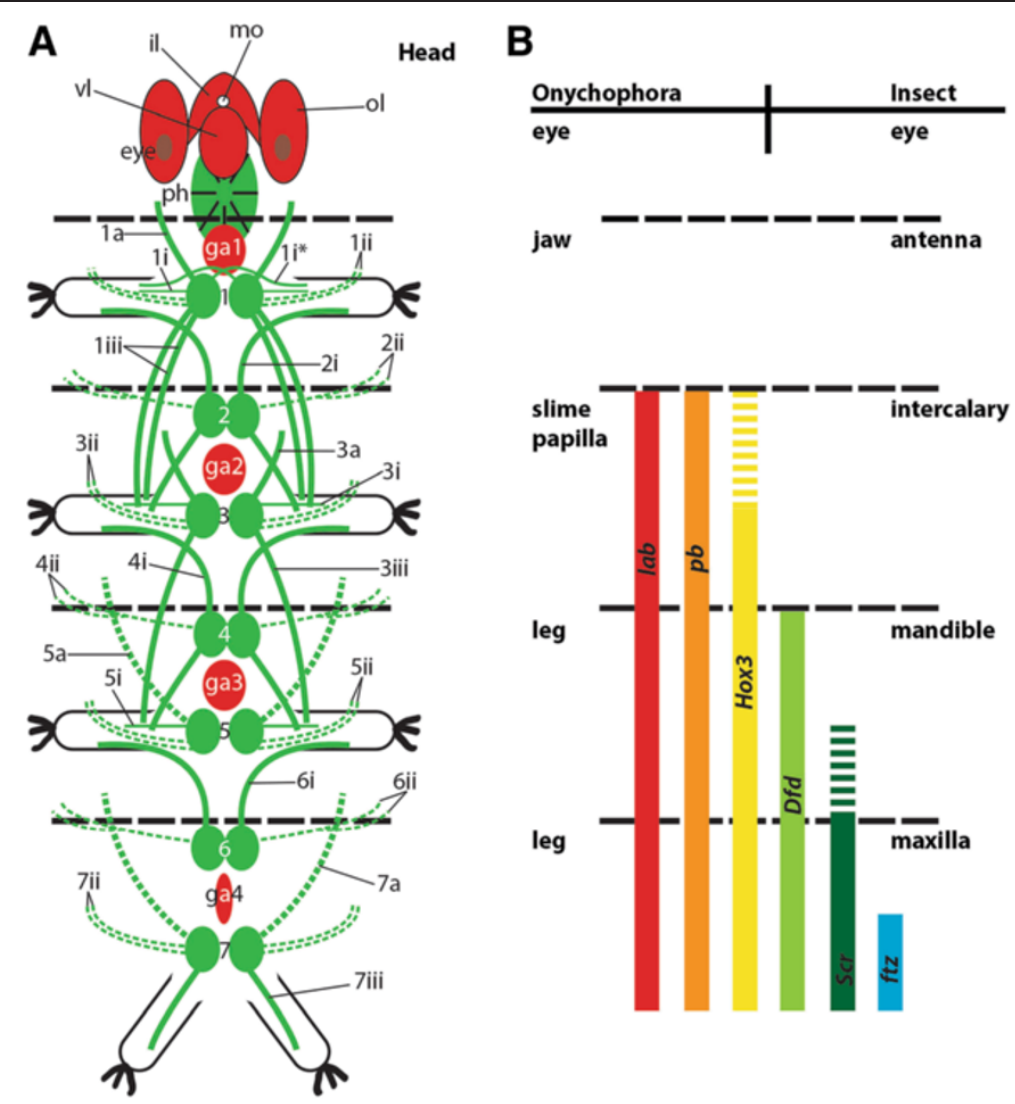

Figure 8 The metameric pattern of $\boldsymbol{H}$. dujardini includes five distinct segment identities. (A) A model $\boldsymbol{H}$. dujardini segmental anatomy based on results of the investigations of ventral nervous and muscle systems presented here. Dashed lines demarcate the hypothesized boundaries between segments. Nervous system structures are depicted in red. Muscle system structures are depicted in green. Ventral attachment sites are numbered. Dashed lines represent muscles that extend dorsally. (B) Hypothesized anterior Hox gene expression domains of the onychophoran-arthropod ancestor based on [63]. The identities of onychophoran segments are provided on the left and the identities of insect segments are provided on the right. Dashed expression domains represent uncertainty in reconstruction of ancestral anterior expression boundaries. The anterior-most expression boundary of the Hox genes is the anterior boundary of the third segment. This pattern in H. dujardini would support direct alignment of tardigrade segments with arthropod and onychophoran segments and would suggest that the tardigrade head is composed of a single segment. Abbreviations: Dfd, Deformed; ftz, fushi tarazu; ga, ganglion; lab, labial; il, inner lobe; mo, mouth; ol, outer lobe; ph, pharyngeal bulb; pb, proboscipedia, Scr, Sex combs reduced; vl, ventral lobe.

have been identified in the eutardigrade species Halobiotus crispae [17]. Corresponding structures were not identified in two species of Macrobiotus [15,35], which are also eutardigrades. Based on these studies, it was suggested that a subesophogeal ganglion is not part of the tardigrade brain $[15,35]$. While we did not detect a discrete subesophogeal ganglion in $H$. dujardini, we suggest that the ventral brain lobe of this species can be homologized with the ventral brain structures of $\mathrm{H}$. crispae [17]. The structure that we identified in $H$. dujardini as the ventral brain lobe (Figures 2B, 3A, 3D) is horseshoe-shaped. The ventral body of this structure is found in a position that corresponds to the position of the subesophogeal ganglion in H. crispae, while the branches of the ventral lobe extend around either side of the buccal tube (Figure 2C) to positions that correspond to the position of the paired ventral lobes in H. crispae. Based on similarities in size and position of the subesophogeal ganglion and ventral lobes of $H$. crispae to the ventral body and branches of the ventral lobe in $H$. dujardini, we propose that these structures are directly homologous. Based on our homology assignments and the fact that $H$. dujardini is more closely related to Macrobiotus than to $H$. crispae [55], we conclude that the ancestor of Eutardigrada included brain regions homologous to the outer, inner, and ventral brain lobes of $H$. dujardini. The interpretation of the brain anatomy of the heterotardigrade Actinarctus doryphorus by Persson et al. [18] is consistent with their interpretation of $\mathrm{H}$. crispae. In this case, we interpret the ventral lobe of $H$. dujardini as homologous to the subesophogeal ganglion and third brain lobe in A. doryphorus. The fact that $H$. dujardini and $A$. doryphorus span the basal split of Tardigrada suggests that the tardigrade ancestor also had a brain exhibiting this architecture. 
Additional studies are needed to resolve the debate concerning the morphology of the ventral brain component of tardigrades.

While an understanding is emerging of how the brains of different tardigrade species relate to each other, the question remains of how tardigrade brains relate to the brains of other panarthropods. The tripartite hypothesis is based on the number of brain lobes and commissures found in the tardigrade brain, which are roughly consistent with a brain composed of three ganglia, if each pair of lobes connected by a commissure defines a ganglion. In further support of the tripartite hypothesis, Persson et al. [18] pointed out that differences in the number of sensory appendages found on the heads of arthrotardigrades compared to the leg-bearing segments is consistent with a head composed of multiple segments. However, Zantke et al. [35] noted that the protocerebrum of arthropods is itself composed of multiple lobes, suggesting that brain lobes do not indicate segmental status. Likewise, Mayer et al. [15] noted that a single ganglion can have more than one commissure. Approaches using data other than morphology may be necessary to reveal the relationship of the tardigrade brain to that of other panarthropods (see below).

As with brain anatomy, there are disagreements among various studies regarding nervous system architecture in the leg-bearing segments. However, phylogenetic reconstructions allow us to unambiguously interpret plesiomorphic conditions for Tardigrada. For instance, Zantke et al. [35] did not identify ventral commissures in the ganglia of leg bearing segments of M. hufelandi. However, ventral commissures were identified in the ganglia of both heterotardigrade $[18,36,37]$ and eutardigrade species [16,17], including $M$. harmsworthi [15], suggesting that ganglionic ventral commissures are ancestral for tardigrades. In this respect, $H$. dujardini appears to retain the ancestral condition. Interestingly, Mayer et al. [16] detected extra-ganglionic interpedal commissures in H. dujardini, M. harmsworthi, and Paramacrobiotus richtersi using combined anti-tyrosinated and anti-acetylated $\alpha$-tubulin immunolabeling. These structures are also present in E. testudo [36]. Presence of these structures in taxa that span the basal split of Tardigrada argues that they are ancestral components of tardigrade nervous systems [16].

We did not detect obvious morphological differences between the ganglia of the first three leg-bearing segments. However, these three ganglia are noticeably wider than the ganglion of the posterior-most segment (Figure 3A). Segmental differences in nervous system anatomy have also been reported for other tardigrade species. As in $H$. dujardini, the fourth ganglion of Halobiotus crispae is the most morphologically distinct. In this species, the first three trunk ganglia each have two transverse commissures, while the fourth trunk ganglion has only one [17]. Detailed investigations of ganglion morphology are also available for two species of Macrobiotus. Zantke et al. [35] reported differences in the number of cells composing each ganglion. In this species, the anterior-most ganglion was consistently composed of the most cells and the posterior-most ganglion was consistently composed of the fewest cells, while the $2^{\text {nd }}$ and $3^{\text {rd }}$ ganglia exhibited similar intermediate cell numbers. As in $H$. dujardini, in M. harmsworthi, the first three ganglia are similar in size, while the posterior-most ganglion is much smaller [16]. Therefore, in terms of size, is seems likely that a differentiated fourth ganglion is the ancestral condition for Eutardigrada. Ganglion morphology has been investigated in more detail in $M$. harmsworthi using RFamide immunostaining, which labels a class of neuropeptides. This approach revealed distinct and consistent morphological differences between all segmental ganglia [15]. Schulze and Schmidt-Rhaesa [36] showed that the first and fourth ganglia are narrower than the second and third in E. testudo, a heterotardigrade species. This suggests that a differentiated fourth ganglion is ancestral for Tardigrada, and raises the possibility that the first ganglion was also differentiated. The functional significance, if any, for different sizes among ganglia remains unclear.

\section{Muscle system anatomy of $H$. dujardini and its relationship to that of other tardigrade species}

Muscle anatomy has been investigated in two heterotardigrade species, Batillipes bullacaudatus and Echiniscus testudo [39], and six eutardigrade species, an unidentified Hypsibius species [54], H. crispae [53], Milnesium tardigradum [30,54], Bertolanius volubilis [39], Dactylobiotus parthenogeneticus [39], and Richtersius coronifer [56]. These studies illuminate highly conserved aspects of segmental muscle patterns across tardigrade species (see [33] for reconstructions of ancestral muscle anatomies). For example, the head of all tardigrades, including H. dujardini (Figure 6A), includes muscles associated with the stylets. Interestingly, the stylets and stylet glands of the pharyngeal apparatus have been proposed to be serially homologous to the claws and claw glands of tardigrade legs, suggesting that the stylets and associated structures may be derived from ancestral legs of the head [20]. This is supported by the fact that only leg muscles and stylet muscles exhibit cross-striation patterns in the tardigrade species $H$. crispae [53]. We did not investigate whether these two muscle types are striated in H. dujardini.

Of particular interest is the arrangement of ventral attachment sites in the leg-bearing segments among tardigrade species. Seven attachment sites is a conserved feature of tardigrades. In both Hypsibius (Figure 4A) and M. tardigradum [54] the left and right halves of ventral attachment sites make contact at the midline in even-numbered 
attachment sites, but not in odd-numbered attachment sites. These two taxa span the basal eutardigrade node in a recent molecular phylogeny [55], suggesting that they likely inherited this pattern from the eutardigrade common ancestor. Two exceptions to this rule are found within Eutardigrada. First, in H. crispae, the left and right halves make contact at every ventral median attachment site except for site 5 [53]. Second, in $R$. coronifer, the left and right halves of attachment site 1 make contact at the midline [56]. Whether or not the pattern revealed in $H$. dujardini and $M$. tardigradum exists among heterotardigrade species has not been reported, leaving open the question of whether this is the ancestral state for Tardigrada.

The first leg-bearing segment differs from more posterior ones in having only a single ventral median attachment site in both $H$. dujardini (Figures 4A and 8A) and other tardigrade species [39,53,54]. A thin muscle crosses the midline of attachment site 1 and extends into the contralateral leg in both eutardigrades, including $H$. dujardini (Figures $4 \mathrm{~A}$ and $8 \mathrm{~A}$ ), and heterotardigrades [39]; as a rule, muscles do not cross the midline at more posterior ventral attachment sites in tardigrade species investigated. The only exception to this rule is found in $H$. crispae; in this species, the posteriorly directed muscles extending from attachment site 5 cross the midline [53]. More posterior segments usually possess two attachment sites each. The exception to this rule is the heterotardigrade species B. bullacaudatus, in which ventral attachment site 3 is divided into two separate nodes, resulting in three attachment sites in the second segment [39]. However, because the heterotardigrade species $E$. testudo has a single attachment site 3 [39], like eutardigrades, including $H$. dujardini (Figures 4A and $8 \mathrm{~A}$ ), it is inferred that ancestrally, in Tardigrada, legbearing segments 2-4 each contained two attachment sites. Ventral attachment site 2 is identical to attachment site 4 in eutardigrade species investigated [39,53,54], including $H$. dujardini (Figures 4A and 8A). This pattern is also found in the heterotardigrade E. testudo [39], suggesting that it is the ancestral condition for tardigrades. Interestingly, in the heterotadigrade species B. bullacaudatus, ventral attachment site 2 is morphologically distinct from attachment site 4 [39]. As with their paired $3^{\text {rd }}$ ventral attachment sites, this is likely a derived condition. Additional investigations of heterotardigrade species should be performed to test the robustness of these reconstructions of ancestral muscle anatomy.

With the exception of B. bullacaudatus, in all tardigrades investigated, including $H$. dujardini (Figures 4A and $8 \mathrm{~A}$ ), ventral attachment site 3 resembles attachment site $5[39,53,54]$. However, in eutardigrade species, including $H$. dujardini, attachment site 3 includes a muscle band that extends to the base of the third leg; a corresponding muscle is lacking in attachment site 5 [39,53,54]. In all species investigated, ventral median attachment sites 6 and 7 show distinct architectures compared to more anterior ventral attachment sites; these sites also exhibit variation between species, making it difficult to infer their ancestral morphologies. Finally, as with $H$. dujardini leg muscle anatomy (Figure 7), muscle anatomy of the fourth leg pair is always the most differentiated in other tardigrade species; these legs generally possess fewer muscles than other legs $[39,53,54]$. Of the more anterior leg pairs, the anatomy of the first leg pair is the most differentiated (Figure 7A, B) $[39,53,54]$. While the lateral musculature is very similar among the anterior three leg pairs, the first leg pair lack iii muscles, which attach to the anterior bases of the $2^{\text {nd }}$ and $3^{\text {rd }}$ leg pairs (Figures $7 \mathrm{~A}$ and $8 \mathrm{~A}$ ).

\section{The segmental regionalization pattern of tardigrades and its relationship to that of other panarthropods}

Although the relationship among the segments of tardigrades is not a contentious issue, the relationship of their segments to those of other panarthropods remains problematic. It seems unlikely that investigations of the neural anatomies of additional species will allow distinguishing between the protocerebral and tripartite hypotheses of brain anatomy. These hypotheses are based on different evolutionary interpretations of very similar morphologies; i.e., additional data are unlikely to sway opinions in either direction. This debate may seem familiar; it is quite similar to the historic debate among arthropod biologists concerning the homology of head segments of Chelicerata to head segments of mandibulate arthropods (see [30]). In this case, expression patterns of Hox genes revealed a conserved pattern of head segmentation across arthropods [40-42,57-62]. Moreover, expression patterns of Hox genes in onychophorans have revealed the relationship of their head segments to those of arthropods [51,63]. One conclusion of these studies is that the anterior expression boundaries of anterior Hox genes evolved in an ancestor of arthropods + onychophorans and have been highly conserved.

These conserved expression patterns suggest that the developmental mechanisms that differentiate the anterior segments of arthropods already existed in the shared ancestor of Onychophora and Arthropoda, even though the ancestor is thought to have exhibited homonomous segments [32,33]. Why would the ancestor of these two lineages, which is hypothesized to have lacked obvious morphological distinctions between segments, already possess the developmental machinery to produce morphologically distinct anterior segments? Our model of tardigrade segmentation presents the possibility that the muscle system and nervous system had already evolved distinct segmental morphologies in the panarthropod ancestor, long before the evolution of conspicuously 
different segment morphologies and tagmatization patterns found in extant arthropods and onychophorans. Therefore, the developmental mechanisms differentiating the anterior segments of both onychophorans and arthropods may have been responsible for differentiation of the nervous system and muscular system in the anterior segments of the panarthropod ancestor, explaining the similarity of these mechanisms between Onychophora and Arthropoda. If so, arthropod-like segmental expression patterns of Hox genes might explain the subtle segmental differentiation of tardigrades. Functional and expression studies of the Hox genes in $H$. dujardini would illuminate ancestral anterior segment patterning mechanisms and whether these genes underlie the production of segmental differentiation in tardigrades. Especially relevant to arguments about the segmental composition of the tardigrade head, the discovery of conserved arthropod-like segmental Hox expression patterns in $H$. dujardini would suggest that the tardigrade head is composed of a single segment that is directly homologous to the arthropod and onychophoran protocerebral segment (Figure 8B).

\section{Conclusions}

Tardigrades are an enigmatic group closely related to arthropods with a unique metameric pattern consisting of a head and four leg-bearing segments. We provide a detailed description of segmental differentiation in the emerging developmental genetic model tardigrade $H$. dujardini (Figure 8A), based on examination of muscle and nervous system anatomy. Although morphological differentiation between some segments is subtle, these subtle differences are shared with distantly related tardigrades. This provides evidence that the ancestor of tardigrades already possessed segmental differentiation. Furthermore, unique segmental identities are present in the anterior segments of onychophorans and arthropods, and segmental differentiation in these taxa is the product of shared developmental mechanisms (Figure 8B). Together, these data suggest that the ancestor of Panarthropoda already had a series of differentiated anterior segments, and lead to the hypothesis that the developmental mechanisms producing segmental differentiation predate the divergence of the three panarthropod lineages.

\section{Materials and methods}

\section{Maintaining tardigrade cultures}

Our line of $H$. dujardini was originally collected from a benthic pond sample in Darcy Lever, Bolton, Lancashire, England [43], and has been maintained in culture since 1987. For this study, tardigrade cultures were maintained in $60 \mathrm{~mm}$ polystyrene petri dishes in $12.5 \mathrm{ml}$ of Poland Spring brand water. Tardigrades were fed a diet of Chlorococcum sp. algae. Cultures were inspected weekly, and fresh water was added to offset evaporation. Subcultures were prepared at least once a month and parent cultures were allowed to expire. We have maintained our $H$. dujardini cultures for over 3 years with this method.

\section{Specimen relaxation and fixation}

Juvenile and reproductively mature $H$. dujardini specimens were collected for this study. Tardigrades tend to contract their muscles during the fixation process, making it difficult to interpret anatomy. To prevent contraction, specimens were relaxed in carbonated water for 1 hour before fixation. At the end of this hour, the bodies of specimens appeared turgid and their appendages were completely extended. Specimens were fixed in $4 \%$ EM Grade Paraformaldehyde (Electron Microscopy Sciences) in PB-Triton (1X phosphate-buffered saline, $0.1 \%$ Triton $\mathrm{X}-100, \mathrm{pH} 7.4$ ) for 15 minutes at room temperature. Specimens were used immediately for staining of the muscle or nervous systems, as described below.

\section{Anti- $\beta$-tubulin immunostaining}

We stained the nervous system of $H$. dujardini using a monoclonal anti- $\beta$-tubulin antibody (E7, Developmental Studies Hybridoma Bank) [64], following a slightly modified version of a protocol previously used to stain $\alpha$-tubulin in a different tardigrade species [29]. Fixation was followed by three 5 minute and four 30 minute rinses in PB-Triton. At this point, the specimens were in a $1.5 \mathrm{ml}$ microcentrifuge tube filled with $1 \mathrm{ml}$ of PB-Triton. Specimens were then permeabilized by sonicating them for 25 seconds with an FS30 sonicater (Fisher Scientific) filled with $850 \mathrm{ml}$ of water. This was followed by three 10 minute and four 30 minute washes in PB-Triton with $0.2 \%$ Bovine Serum Albumin and two 30 minute washes in PB-Triton with 5\% normal goat serum (NGS). Specimens were then incubated at $4^{\circ} \mathrm{C}$ overnight in a 1:100 dilution of $\beta$-tubulin antibody in PB-Triton + NGS. This incubation period was followed by three 5 minute and four 30 minute washes in PBTriton. Specimens were then washed twice for 30 minutes in PB-Triton + NGS. This was followed by an overnight incubation at $4^{\circ} \mathrm{C}$ in a 1:200 dilution of a goat anti-mouse Cy3-conjugated secondary antibody (Jackson ImmunoResearch). This incubation period was followed by three $5 \mathrm{mi}$ nute and six 30 minute washes in PB-Triton.

\section{Phalloidin staining}

We stained muscle cells with Oregon Green 488 phalloidin (Molecular Probes), which binds actin filaments, following a slightly modified version of the protocol used by Halberg et al. [53]. Fixation was followed by four 15 minute and one overnight wash in PB-Triton with $0.1 \% \mathrm{NaN}_{3}$. Specimens were then incubated at room temperature for $27 \mathrm{hrs}$ in a 1:40 dilution of phalloidin in PB-Triton with $0.1 \%$ $\mathrm{NaN}_{3}$. Following phalloidin incubation, specimens were rinsed three times for 5 minutes in PB-Triton. 


\section{Specimen preparation, imaging, and analysis}

The scanning electron micrograph protocol was previously published [44]. For other imaging techniques, specimens were taken through a glycerol series, which included 1 hour washes in 5\%,10\%,20\%, and 40\% glycerol in PB-Triton. Specimens were then mounted on slides in Fluoromount-G (SouthernBiotech) with $1.5 \mu \mathrm{g} / \mathrm{ml} \mathrm{4'}$, 6diamidino-2-phenylindole (DAPI) added to counterstain nuclei. Differential interference contrast (DIC) micrographs were taken on a Zeiss Axioskop or a Nikon A1R Spectral Confocal Microscope. Z-series of phalloidin $(\mathrm{N}=8)$ and anti- $\beta$-tubulin $(\mathrm{N}=3)$ stained specimens were taken on a Nikon A1R Spectral Confocal Microscope. Z-series for 2 additional anti- $\beta$-tubulin stained specimens were acquired with a Nikon Eclipse Ti spinning disk confocal microscope. Figi [65-67] was used for image analysis and processing. The Merge function in Figi was used to produce images showing data from multiple confocal channels. Depth-coded images were produced using the Temporal-Color Code function in Figi. Maximum projection images were produced using the maximum intensity option of the Z Project function in Figi. Image levels were adjusted in Adobe Photoshop CS4.

\section{Additional file}

Additional file 1: Figure S1. Autofluorescence of algal gut content The gut is outlined. (A) Unstained specimen with algal gut content. The algal gut content exhibits a high degree of autofluorescence. (B) DIC image of the same specimen. Algae can be seen in the gut.

\section{Competing interests}

The authors declare that they have no competing interests.

\section{Authors' contributions}

FWS and ELJ conceived of the study, designed the study, and edited the manuscript. FWS performed all wet lab work, collected data, analyzed data and wrote the first draft of the manuscript. Both authors have read and approved the manuscript.

\begin{abstract}
Acknowledgments
We thank Carl D. Schlichting, David L. Wagner, Steven Q. Irvine, and two anonymous reviewers for valuable comments on the manuscript. We are incredibly grateful to Bob Goldstein for providing a H. dujardini culture and SEM images for this study. Vicky Nelson assisted in acquisition of SEMs. Karolina Fučíková provided advice for culturing algae. Louise Lewis provided culturing media for algae. Carol Norris of the University of Connecticut Confocal Microscopy Facility provided training and assistance on the confocal scopes used during this study. This research was partially funded by the following awards to FWS: 1.) Betty Decoursey Endowment and the James A. Slater Endowment Fund of the University of Connecticut Ecology and Evolutionary Biology Department and the Connecticut State Museum of Natural History; 2.) a Society for the Study of Evolution (SSE) Rosemary Grant Graduate Student Research Award; 3.) an Extraordinary Expense Award from the University of Connecticut Graduate School.
\end{abstract}

Received: 27 March 2014 Accepted: 3 September 2014

Published online: 17 September 2014

\section{References}

1. Horikawa DD, Sakashita T, Katagiri C, Watanabe M, Kikawada T, Nakahara Y, Hamada N, Wada S, Funayama T, Higashi S, Kobayashi Y, Okuda T, Kuwabara M:
Radiation tolerance in the tardigrade Milnesium tardigradum. Int J Radiat Biol 2006, 82:843-848.

2. Jönsson Kl, Rabbow E, Schill RO, Harms-Ringdahl M, Rettberg P: Tardigrades survive exposure to space in low earth orbit. Curr Biol 2008, 18:R729-R731.

3. Hengherr S, Worland MR, Reuner A, Brümmer F, Schill RO: High temperature tolerance in anhydrobiotic tardigrades is limited by glass transition. Physiol Biochem Zool 2009, 82:749-755.

4. Seki K, Toyoshima M: Preserving tardigrades under pressure. Nature 1998 395:853-854.

5. Rebecchi L, Altiero T, Guidetti R, Cesari M, Bertolani R, Negroni M, Rizzo MA: Tardigrade resistance to space effects: first results of the experiment of LIFE - TARSE mission on FOTON-M3 (September 2007). Astrobiology 2009, 9:581-591

6. Persson D, Halberg KA, Jørgensen A, Ricci C, Møbjerg N, Kristensen RM: Extreme stress tolerance in tardigrades: Surviving space conditions in low earth orbit. J Zool Syst Evol Res 2011, 49:90-97.

7. Halberg KA, Persson D, Ramløv H, Westh P, Kristensen RM, Møbjerg N: Cyclomorphosis in Tardigrada: adaptation to environmental constraints. J Exp Biol 2009, 212:2803-2811.

8. Jönsson Kl, Harms-Ringdahl M, Torudd J: Radiation tolerance in the eutardigrade Richtersius coronifer. Int J Radiat Biol 2005, 81:649-656.

9. Maas A, Waloszek D: Cambrian derivatives of the early arthropod stem lineage, pentastomids, tardigrades and lobopodians-an 'Orsten' perspective. Zool Anz 2001, 240:451-459.

10. Brusca RC, Brusca GJ: From the emergence of the arthropods: onychophorans, tardigrades, trilobites, and the arthropod bauplan. In Invertebrates. 2nd edition. Sunderland MA: Sinaur Associates; 2003:461-510.

11. Kinchin IA: The Biology of Tardigrades. London: Portland Press Limited; 1994

12. Dewel RA, Dewel WC: The brain of Echiniscus viridissimus Peterfi, 1956 (Heterotardigrada): a key to understanding the phylogenetic position of tardigrades and the evolution of the arthropod head. Zool J Linn Soc 1996, 116:35-49.

13. Kristensen RM, Higgins RP: A new family of Arthrotardigrada (Tardigrada: Heterotardigrada) from the Atlantic coast of Florida, USA. Trans Am MicrosC Soc 1984, 103:295-311.

14. Kristensen RM, Higgins RP: Revision of Styraconyx (Tardigrada: Halechiniscidae) with descriptions of two new species from Disko Bay, West Greenland. Smithson Contrib Zool 1984, 391:1-40.

15. Mayer G, Kauschke S, Rüdiger J, Stevenson PA: Neural markers reveal a one-segmented head in tardigrades (water bears). PLOS ONE 2013, 8:e59090.

16. Mayer G, Martin C, Rudiger J, Kauschke S, Stevenson P, Poprawa I, Hohberg K, Schill RO, Pflüger $\mathrm{H}-J$, Schlegel M: Selective neuronal staining in tardigrades and onychophorans provides insights into the evolution of segmental ganglia in panarthropods. BMC Evol Biol 2013, 13:230.

17. Persson DK, Halberg KA, Jorgensen A, Mobjerg N, Kristensen RM: Neuroanatomy of Halobiotus crispae (Eutardigrada: Hypsibiidae): tardigrade brain structure supports the clade Panarthropoda. J Morphol 2012, 273:1227-1245.

18. Persson DK, Halberg KA, Jørgensen A, Møbjerg N, Kristensen RM: Brain anatomy of the marine tardigrade Actinarctus doryphorus (Arthrotardigrada). J Morphol 2014, 275:173-190.

19. Manton SM: The Arthropoda: Habits, Functional Morphology and Evolution. Oxford: Clarendon Press; 1977.

20. Nielsen C: Animal Evolution: Interrelationships of the Animal Phyla. New York: Oxford University Press; 2001

21. Budd GE: The morphology of Opabinia regalis and the reconstruction of the arthropod stem-group. Lethaia 1996, 29:1-14.

22. Budd GE: Tardigrades as 'stem-group arthropods': the evidence from the Cambrian fauna. Zool Anz 2001, 240:265-279.

23. Budd GE: Why are arthropods segmented? Evol Dev 2001, 3:332-342.

24. Campbell LI, Rota-Stabelli O, Edgecombe GD, Marchioro T, Longhorn SJ, Telford MJ, Philippe H, Rebecchi L, Peterson KJ, Pisani D: MicroRNAs and phylogenomics resolve the relationships of Tardigrada and suggest that velvet worms are the sister group of Arthropoda. Proc Natl Acad Sci USA 2011, 108:15920-15924.

25. Legg DA, Sutton MD, Gregory DE: Arthropod fossil data increase congruence of morphological and molecular phylogenies. Nat Commun 2013, 4:2485.

26. Dewel RA, Dewel WC: The place of tardigrades in arthropod evolution. In Arthropod Relationships. Edited by Fortey RA, Thomas RH. London: Chapman and Hall; 1997:109-123. 
27. Ma X, Edgecombe GD, Legg DA, Hou X: The morphology and phylogenetic position of the Cambrian lobopodian Diania cactiformis. J Syst Palaeontol 2014, 12:445-457.

28. Angelini DR, Kaufman TC: Comparative developmental genetics and the evolution of arthropod body plans. Annu Rev Genet 2005, 39:95-119.

29. Fusco G, Minelli A: Arthropod segmentation and tagmosis. In Arthropod Biology and Evolution. Edited by Fusco G, Minelli A. Berlin: Springer-Verlag; 2013:197-221.

30. Scholtz G, Edgecombe GD: The evolution of arthropod heads: reconciling morphological, developmental and palaeontological evidence. Dev Genes Evol 2006, 216:395-415.

31. Mayer G, Whitington PM, Sunnucks P, Pfluger HJ: A revision of brain composition in Onychophora (velvet worms) suggests that the tritocerebrum evolved in arthropods. BMC Evol Biol 2010, 10:255.

32. Ma X, Hou X, Bergstrom J: Morphology of Luolishania longicruris (Lower Cambrian, Chengjiang Lagerstatte, SW China) and the phylogenetic relationships within lobopodians. Arthropod Struct Dev 2009, 38:271-291.

33. Ou Q, Degan S, Mayer G: Cambrian lobopodians and extant onychophorans provide new insights into early cephalization in Panarthropoda. Nat Commun 2012, 3:1261.

34. Kristensen RM: The first record of cyclomorphosis in Tardigrada based on a new genus and species from Arctic meiobenthos. J Zool Syst EvolutForsch 1982, 20:249-270.

35. Zantke J, Wolff C, Scholtz G: Three-dimensional reconstruction of the central nervous system of Macrobiotus hufelandi (Eutardigrada, Parachela): Implications for the phylogenetic position of Tardigrada. Zoomorphology 2008, 127:21-36.

36. Schulze C, Schmidt-Rhaesa A: The architecture of the nervous system of Echiniscus testudo (Echiniscoidea, Heterotardigrada). J Limnol 2013, 72:44-53.

37. Schulze C, Neves RC, Schmidt-Rhaesa A: Comparative immunohistochemical investigation on the nervous system of two species of Arthrotardigrada (Heterotardigrada, Tardigrada). Zool Anz 2014, 253:225-235.

38. Bitsch J, Bitsch C: The tritocerebrum and the clypeolabrum in mandibulate arthropods: segmental interpretations. Acta Zool-Stockholm 2010, 91:249-266.

39. Marchioro T, Rebecchi L, Cesari M, Hansen JG, Viotti G, Guidetti R: Somatic musculature of Tardigrada: phylogenetic signal and metameric patterns. Zool J Linn Soc Lond 2013, 169:580-603.

40. Damen WGM, Hausdorf M, Seyfarth E-A, Tautz D: A conserved mode of head segmentation in arthropods revealed by the expression pattern of Hox genes in a spider. Proc Natl Acad Sci USA 1998, 95:10665-10670.

41. Jager M, Murienne J, Clabaut C, Deutsch J, Le Guyader H, Manuel M: Homology of arthropod anterior appendages revealed by Hox gene expression in a sea spider. Nature 2006, 411:506-508.

42. Telford MJ, Thomas RH: Expression of homeobox genes shows chelicerate arthropods retain their deutocerebral segment. Proc Natl Acad Sci USA 1998, 95:10671-10675.

43. Gabriel WN, McNuff R, Patel SK, Gregory TR, Jeck WR, Jones CD, Goldstein B: The tardigrade Hypsibius dujardini, a new model for studying the evolution of development. Dev Biol 2007, 312:545-559.

44. Gabriel WN, Goldstein B: Segmental expression of Pax3/7 and Engrailed homologs in tardigrade development. Dev Genes Evol 2007, 217:421-433.

45. Smith FW: Conservation and Diversification of Appendage Identity Specification Mechanisms along the Anteroposterior and Proximodistal Axes in Panarthropoda, PhD dissertation. Connecticut: University of Connecticut, Department of Ecology and Evolutionary Biology; 2013.

46. Tenlen JR, McCaskill S, Goldstein B: RNA interference can be used to disrupt gene function in tardigrades. Dev Genes Evol 2013, 223:1-11.

47. Budd GE, Telford MJ: The origin and evolution of arthropods. Nature 2009, 457:812-817.

48. Grenier JK, Garber TL, Warren R, Whitington PM, Carroll S: Evolution of the entire arthropod Hox gene set predated the origin and radiation of the onychophoran/arthropod clade. Curr Biol 1997, 7:547-553.

49. Shubin N, Tabin C, Carroll S: Fossils, genes and the evolution of animal limbs. Nature 1997, 388:639-648.

50. Snodgrass RE: Principles of Insect Morphology. Ithaca: Cornell University Press; 1935

51. Eriksson BJ, Tait NN, Budd GE, Janssen R, Akam M: Head patterning and Hox gene expression in an onychophoran and its implications for the arthropod head problem. Dev Genes Evol 2010, 220:117-122.
52. Eriksson BJ, Samadi L, Schmid A: The expression pattern of the genes engrailed, pax6, otd and six3 with special respect to head and eye development in Euperipatoides kanangrensis Reid 1996 (Onychophora: Peripatopsidae). Dev Genes Evol 2013, 223:237-246.

53. Halberg KA, Persson D, Mobjerg N, Wanninger A, Kristensen RM: Myoanatomy of the marine tardigrade Halobiotus crispae (Eutardigrada: Hypsibiidae). J Morphol 2009, 270:996-1013.

54. Schmidt-Rhaesa A, Kulessa J: Muscular architecture of Milnesium tardigradum and Hypsibius sp. (Eutardigrada, Tardigrada) with some data on Ramazottius oberhaeuseri. Zoomorphology 2007, 126:265-281.

55. Guil N, Giribet G: A comprehensive molecular phylogeny of tardigradesadding genes and taxa to a poorly resolved phylum-level phylogeny. Cladistics 2012, 28:21-49.

56. Halberg KA, Jorgensen A, Mobjerg N: Desiccation tolerance in the tardigrade Richtersius coronifer relies on muscle mediated structural reorganization. PLoS One 2013, 8(12):e85091.

57. Abzhanov A, Kaufman TC: Homeotic genes and the arthropod head: expression patterns of the labial, proboscipedia, and Deformed genes in crustaceans and insects. Proc Natl Acad Sci USA 1999, 96:10224-10229.

58. Damen WGM, Tautz D: A Hox class 3 orthologue from the spider Cupiennius salei is expressed in a Hox-gene-like fashion. Dev Genes Evol 1998, 208:586-590.

59. Hughes $C L$, Kaufman TC: Hox genes and the evolution of the arthropod body plan. Evol Dev 2002, 4:459-499.

60. Janssen R, Damen WG: The ten Hox genes of the millipede Glomeris marginata. Dev Genes Evol 2006, 216:451-465.

61. Manuel M, Jager M, Murienne J, Clabaut C, Le Guyader H: Hox genes in sea spiders (Pycnogonida) and the homology of arthropod head segments. Dev Genes Evol 2006, 216:481-491.

62. Sharma PP, Schwager EE, Extavour CG, Giribet G: Hox gene expression in the harvestman Phalangium opilio reveals divergent patterning of the chelicerate opisthosoma. Evol Dev 2012, 14:450-463.

63. Janssen R, Eriksson BJ, Tait NN, Budd GE: Onychophoran Hox genes and the evolution of arthropod Hox gene expression. Front Zool 2014, 11:22.

64. Chu DT, Klymkowsky MW: The appearance of acetylated alpha-tubulin during early development and cellular differentiation in Xenopus. Dev Biol 1989, 1989(136):104-117.

65. Abràmoff MD, Magalhães PJ, Ram SJ: Image processing with ImageJ. Biophoton Int 2004, 11:36-42.

66. Rasband WS: 1997-2012. Image J. Bethesda, Maryland, USA: U.S. National Institutes of Health; imagej.nih.gov/ij/.

67. Schneider CA, Rasband WS, Eliceiri KW: NIH image to ImageJ: 25 years of image analysis. Nat Methods 2012, 9:671-675.

doi:10.1186/s12983-014-0066-9

Cite this article as: Smith and Jockusch: The metameric pattern of Hypsibius dujardini (Eutardigrada) and its relationship to that of other panarthropods. Frontiers in Zoology 2014 11:66.

\section{Submit your next manuscript to BioMed Central and take full advantage of:}

- Convenient online submission

- Thorough peer review

- No space constraints or color figure charges

- Immediate publication on acceptance

- Inclusion in PubMed, CAS, Scopus and Google Scholar

- Research which is freely available for redistribution 